\title{
Accuracy of Delivery and Effects on Absolute Humidity of Low Tidal Volume by ICU Ventilators
}

\author{
Barbara Moro MD, Loredana Baboi PhD, Hodane Yonis MD, Fabien Subtil PhD, \\ Bruno Louis $\mathrm{PhD}$, and Claude Guérin $\mathrm{MD} \mathrm{PhD}$
}

\begin{abstract}
BACKGROUND: During extracorporeal membrane oxygenation for ARDS, a range of 1-4 mL/kg predicted body weight tidal volume $\left(V_{T}\right)$ is commonly used. We explored whether such a low $V_{T}$ could be adequately delivered by ICU ventilators, and whether such low $\mathrm{V}_{\mathrm{T}} \mathrm{s}$ prevent the heated humidifier (HH) from reaching the recommended target of $33 \mathrm{mg} / \mathrm{L}$ absolute humidity (AH). METHODS: We attached a lung model to 5 ICU ventilators set in volume controlled mode and body temperature and pressure saturated. We ran 2 protocols over a $100-280 \mathrm{~mL} V_{T}$ range used with adult or neonatal breathing circuit at a breathing frequency (f) of 15 (f15) or 30 (f30) breaths/min. In the first protocol, with the $\mathrm{HH}$ off, $\mathrm{V}_{\mathrm{T}}$ was measured with a dedicated data logger and expressed in body temperature and pressure saturated. The relationships of measured $V_{T}$ to set $V_{T}$ were studied, and the relative error in $V_{T}$ was analyzed within its $10 \%$ boundaries. In the second protocol, the $\mathrm{HH}$ was on, and we measured $\mathrm{AH}$ using a psychrometric method. The relationship of $\mathrm{AH}$ to set $\mathrm{V}_{\mathrm{T}}$ was analyzed with linear regression. RESULTS: For the 5 ventilators used, the slope $(95 \% \mathrm{CI})$ between measured $V_{T}$ versus set $V_{T}$ averaged $0.93(0.92-0.93), 0.93(0.93-0.94), 0.91(0.90-0.91)$, and 0.91 $(0.90-0.91) \mathrm{mL} / \mathrm{mL}$ for adult and neonatal circuits at f15 and f30, respectively $(P<.05$ vs 1 in each instance), indicating a systematic under-delivery of $V_{T}$. The $V_{T}$ relative error fell within the $\pm 10 \%$ accuracy range for only 2 ventilators with adult circuits at f15 and f30. AH increased linearly with $\mathrm{V}_{\mathrm{T}}$. The recommended target of $33 \mathrm{mg} / \mathrm{L} \mathrm{AH}$ was reached with all of the ventilators for the adult circuit at f30 only. The minimum volume that met the recommended threshold for $\mathrm{AH}$ was 100, 150, 190, 160, and $100 \mathrm{~mL}$ for the G5, Carestation, PB980, Servo-U, and V500 ventilators, respectively, at f30. CONCLUSION: Low $V_{T}$ was systematically under-delivered by modern ICU ventilators by roughly 7-9\%. To meet the recommended target of $33 \mathrm{mg} / \mathrm{L} \mathrm{AH}$, adult circuit at f30 should be used. Key words: ARDS; extracorporeal membrane oxygenation; tidal volume; humidification; absolute humidity; accuracy. [Respir Care 2018;63(10):1253-1263. (C) 2018 Daedalus Enterprises]
\end{abstract}

\section{Introduction}

In patients with severe ARDS who require extracorporeal membrane oxygenation (ECMO), appropriate ventilator settings are still debated; however, the overall aim should be to rest the lung. ${ }^{1}$ The median tidal volume $\left(\mathrm{V}_{\mathrm{T}}\right)$ reported in the literature at this setting is $4 \mathrm{~mL} / \mathrm{kg}$ pre-

Drs Moro, Baboi, Yonis, and Guérin are affiliated with Réanimation Médicale, Hôpital de la Croix Rousse, Hospices Civils de Lyon, Lyon, France. Dr Subtil is affiliated with Service de Biostatistique, Hospices Civils de Lyon, Lyon, France and Université Lyon 1, CNRS, Laboratoire de Biométrie et Biologie Evolutive UMR 5558, Villeurbanne, France. Drs Louis and Guérin are affiliated with Institut Mondor de Recherche Biomédicale INSERM 955, Créteil, France. dicted body weight (PBW), with $25 \%$ of patients receiving $2-3 \mathrm{~mL} / \mathrm{kg}$ PBW and $13 \%$ receiving $<2 \mathrm{~mL} / \mathrm{kg}$ PBW. ${ }^{2-4}$ Even though ICU ventilators are high-performance devices, the accuracy of $\mathrm{V}_{\mathrm{T}}$ can be challenged by 2 phenom-

\footnotetext{
Dr Guérin presented a version of this paper at the European Society of Intensive Care Medicine meeting October 2017, Vienna, Austria, and Société de Réanimation de Langue Française meeting January 2018, Paris, France.

Richard Degas at Fisher-Paykel provided the psychrometric device. The authors have disclosed no other conflicts of interest.

Supplementary material related to this paper is available at http:// www.rcjournal.com.
} 
ena with opposite effects. On the one hand, to account for gas compression in the breathing circuit, the ventilator must deliver a greater $\mathrm{V}_{\mathrm{T}}$ than whatever is set to be delivered. Therefore, breathing circuit compliance must be calibrated in the ventilator before use. On the other hand, account for increased volume that accompanies increased temperature once the gas enters the patient, the ventilator must deliver a lower $\mathrm{V}_{\mathrm{T}}$ than whatever is set to be delivered. At the low $\mathrm{V}_{\mathrm{T}}$ values used during ECMO, the volume compressed into the circuit may become meaningful, and the efficiency of the heated humidifier $(\mathrm{HH})$ can be impaired. The amount of minute ventilation is a key determinant for optimal performance of the HH. ${ }^{5}$ Because minute ventilation is equal to $\mathrm{V}_{\mathrm{T}} \times$ breathing frequency (f), $\mathrm{f}$ can be as important as $\mathrm{V}_{\mathrm{T}}$ in the efficiency of the $\mathrm{HH}$. In our practice (unpublished observations) of ECMO in patients with ARDS, low-temperature alarms at the $\mathrm{HH}$ chamber are common and indicate that the device is not able to increase the gas temperature during its travel across the chamber.

The efficiency of the HH is assessed with absolute humidity ( $\mathrm{AH})$, which is the water vapor mass in a given gas volume, and relative humidity $(\mathrm{RH})$, which is the ratio between water vapor mass contained in a certain gas volume at a certain temperature and maximum water vapor mass that this gas volume could contain at the same temperature. Gas delivered by compressed gas ventilators is cold and dry, therefore the use of a humidification device is mandatory in ventilated patients to avoid airway damage and life-threatening complications like endotracheal tube obstruction. When $\mathrm{AH}$ is $44 \mathrm{mg} / \mathrm{L}$ for $100 \% \mathrm{RH}$ at body temperature, it is recommended to maintain $\mathrm{AH} \geq 33 \mathrm{mg} \mathrm{H}_{2} \mathrm{O} / \mathrm{L}$ $(75 \% \mathrm{RH})$ in intubated patients. ${ }^{6}$ The international standard for humidifiers (ISO 8185) specifies that $\mathrm{AH}$ should be at least $33 \mathrm{mg} / \mathrm{L}$ (https://www.sis.se/api/document/preview/ 607054, Accessed June 12, 2018).

To our knowledge, no study has investigated the accuracy of ICU ventilators to deliver low set $\mathrm{V}_{\mathrm{T}}$ and the impact of such low $\mathrm{V}_{\mathrm{T}}$ on $\mathrm{HH}$ efficiency. Our bench study assessed the $V_{T}$ actually delivered by ICU ventilators over a large range of low $\mathrm{V}_{\mathrm{T}}$. Our hypothesis was that delivered $\mathrm{V}_{\mathrm{T}}$ is lower than expected and significantly different between ventilators, as previously found for higher set $\mathrm{V}_{\mathrm{T}}{ }^{7}$ Our second objective was to determine whether such low $\mathrm{V}_{\mathrm{T}}$ can impair the efficiency of the HH in the same ICU ventilators. Our hypothesis was that observed $\mathrm{AH}$ would be $<33 \mathrm{mg} \mathrm{H}_{2} \mathrm{O} / \mathrm{L}$, and that there would be differences

Correspondence: Claude Guérin MD PhD, Service de Réanimation médicale, Hôpital de la Croix Rousse, 103 Grande Rue de la Croix Rousse, 69004 Lyon, France. E-mail claude.guerin@chu-lyon.fr.

DOI: $10.4187 /$ respcare. 06132

\section{QUICK LOOK}

\section{Current knowledge}

Low tidal volumes (1-4 mL/kg predicted body weight) are commonly used in patients with ARDS who receive extracorporeal membrane oxygenation to allow the lung to rest. Our bench study explored whether low tidal volumes were adequately delivered by 5 commonly used ICU ventilators, and whether this could affect the efficiency of heated humidifiers.

\section{What this paper contributes to our knowledge}

We found, testing 5 ICU ventilators, that tidal volume was under-delivered by an average of $7-9 \%$, and that only 2 of these ventilators delivered tidal volume within $10 \%$ accuracy. We found that the recommended $\mathrm{AH}$ threshold of $33 \mathrm{mg} \mathrm{H}_{2} \mathrm{O} / \mathrm{L}$ was delivered with each ventilator only when an adult breathing circuit was used at a breathing frequency of 30 breaths $/ \mathrm{min}$.

between ventilators for the same set minute ventilation. Furthermore, from informal discussions with intensivists using ECMO, we found that neonate breathing circuits are sometimes used in adult patients at low $\mathrm{V}_{\mathrm{T}}$ to minimize the compression of air in the ventilator circuit. Therefore, we tested adult and neonate circuits.

\section{Methods}

Our experimental set-up included 5 ICU ventilators: Carescape R860 (GE Healthcare, Madison, Wisconsin), Evita Infinity V500 (Dräger, Lubeck, Germany), G5 (Hamilton, Bonaduz, Switzerland), PB 980 (Covidien-Medtronic, Boulogne-Billancourt, France), and Servo-U (Maquet, Wayne, New Jersey). The ventilators were actuated in the body temperature and pressure saturated (BTPS) condition and provided by the manufacturers.

We used adult (RT 380, Fisher \& Paykel, Auckland, New Zealand) and neonate (RT 266, Fisher \& Paykel) double heated-wire breathing circuits provided by the manufacturer. We used the MR850 heated humidifier provided by the manufacturer, in combination with the MR290 chamber (Fisher \& Paykel) auto-filled with sterile water (DKB7114, Baxter Healthcare, Zürich, Switzerland). We utilized a pneumatic test lung (Training Test Lung, Michigan Instruments, Grand Rapids, Michigan) set at $20 \mathrm{~mL} / \mathrm{cm}$ $\mathrm{H}_{2} \mathrm{O}$ compliance ${ }^{4}$ and an Rp20 Pneuflo parabolic resistor (Michigan Instruments) to simulate severe ARDS requiring ECMO. We used a pressure transducer (Gabarith PMSET 1DT-XX, Becton Dickinson, Singapore) to measure airway pressure calibrated at room temperature using 
A

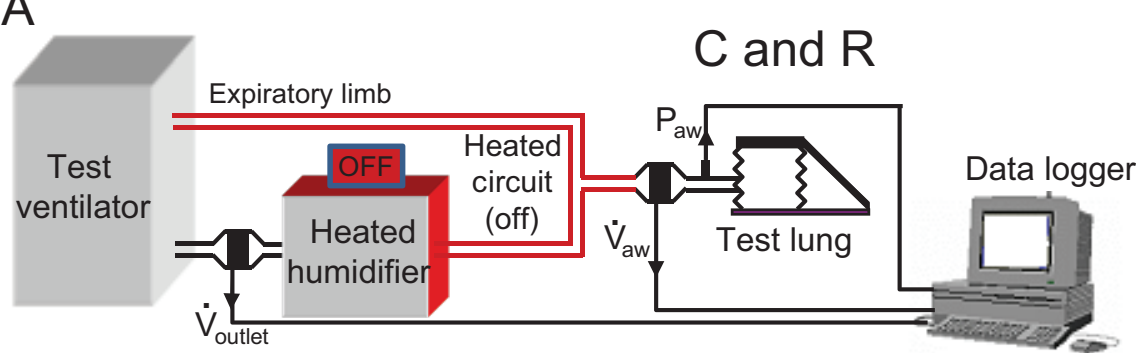

B

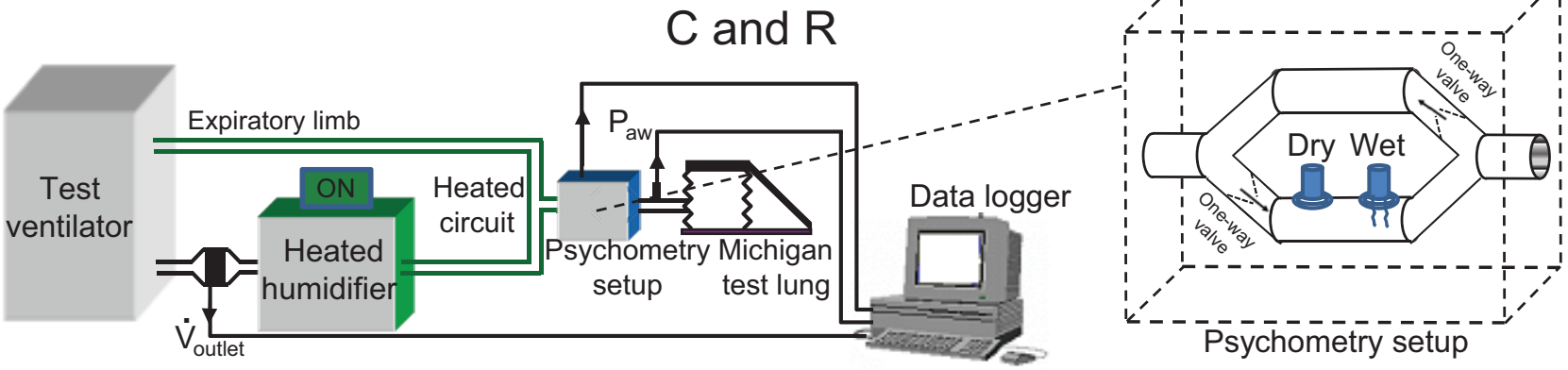

Fig. 1. Experimental set-up used in part $1(\mathrm{~A})$ and part $2(\mathrm{~B})$ of the bench study. $\mathrm{R}=$ resistance; $\mathrm{C}=$ compliance of the test lung; $\mathrm{P}_{\mathrm{aw}}=$ airway pressure; $\mathrm{V}_{\mathrm{aw}}=$ airflow volume.

a manometer $(717$ 1G, Fluke Biomedical, Everett, Washington), ${ }^{8,9}$ and a screen pneumotachometer (3700 series, Hans Rudolph, Shawnee, Kansas) calibrated at room temperature using a precision rotameter (Houdec Glass, Martin Medical, Lyon, France).

We measured $\mathrm{RH}$ and $\mathrm{AH}$ with a psychrometric method that includes a flow splitter and 2 thermal probes (Eurotec, Bologna, Italy) (Fig. 1). We measured room air temperature, pressure, and RH with a Rotronic HygroClip HC2A Hygrometer (Rotronic, Croissy Beaubourg, France). A data logger (MP150, Biopac Systems, Goleta, California) was used to record airway pressure, air flow, and temperature signals.

We conducted this study in the respiratory physiology lab of the ICU at Service de Réanimation Médicale, Hôpital de la Croix Rousse, Hospices Civils de Lyon, Lyon, France. Our study had 2 protocols.

\section{Accuracy of Very Low $V_{T}$ Delivered by ICU Ventilators (Protocol 1)}

The range of very low $\mathrm{V}_{\mathrm{T}}$ selected for this study can be achieved in ICU ventilators by selecting the adult, pediatric, or neonate setting. The adult configuration requires an adult breathing circuit, whereas the pediatric and neonate configurations require a neonate breathing circuit.

Each ventilator was fully checked, with the $\mathrm{HH}$ in place and switched off, according to the procedure described in the manufacturer's user manual. This process included the detection of leaks and the determination of breathing circuit compliance. If available, the algorithm for leak compensation was switched off to unmask the presence of leaks.

Ventilators were set in volume controlled mode with a constant inspiratory flow of $20 \mathrm{~L} / \mathrm{min}, \mathrm{F}_{\mathrm{IO}_{2}} 0.21$, PEEP $12 \mathrm{~cm} \mathrm{H}_{2} \mathrm{O}$, inspiratory pause $0.2 \mathrm{~s}$, and $\mathrm{HH}$ off. We tested a $\mathrm{V}_{\mathrm{T}}$ range of $100-280 \mathrm{~mL}$ in increments of $20 \mathrm{~mL}$. We set the PBW to $70 \mathrm{~kg}$ according to the mean value reported in the Lung Safe study. ${ }^{10}$

A port for airway pressure monitoring was inserted between the Y-piece and the lung model, and a pneumotachometer was inserted between the Y-piece and the airway pressure port (Fig. 1). A second pneumotachometer was inserted at the ventilator outlet to match the set-up in the second protocol (see following section). This allowed us to infer the compliance of the double heated-wire breathing circuits $\left(\mathrm{C}_{\mathrm{c}}\right)$ using $\mathrm{C}_{\mathrm{c}}=\left(\mathrm{V}_{\text {outlet }}-\mathrm{V}_{\mathrm{Y}}\right) / \mathrm{P}_{\text {plateau }}$, where $\mathrm{P}_{\text {plateau }}$ is the pressure plateau during the inspiratory pause. Great care was taken to avoid any leak in the experimental set-up. We verified this by checking the airway pressure stability during an end-inspiratory pause with the complete experimental set-up in place.

Pressure and flow signals were continuously recorded at a sampling rate of $200 \mathrm{~Hz}$. For each set $\mathrm{V}_{\mathrm{T}}$, after 1 min of stabilization, 10 breaths were counted; then the next $\mathrm{V}_{\mathrm{T}}$ was applied and so on, up to $280 \mathrm{~mL} \mathrm{~V} \mathrm{~V}_{\mathrm{T}}$. We 
made this recording first at 30 breaths/min then at 15 breaths/min.

Steps 1-4 were performed with the adult breathing circuit in the adult configuration at the ventilator.

Steps 1-4 were repeated with the neonate breathing circuit in the neonate or pediatric configuration, depending on ventilators. Before testing the neonate breathing circuit, the ventilator set-up was fully checked again with the neonate circuit in place as explained in step 1.

\section{Efficiency of Humidification at Very Low $V_{T}$ (Protocol 2)}

Inspired air conditioning with the MR850 $\mathrm{HH}$ proceeds through the following steps. First is the humidification of air flowing through the chamber during insufflation due to the energy delivered by the heater plate. The temperature of the gas leaving the chamber is set to $37^{\circ} \mathrm{C}$. The encapsulated heater wire maintains the gas temperature and prevents its condensation as it travels through the inspiratory limb of the breathing circuit. The gas temperature at the Y-piece is $40^{\circ} \mathrm{C}$ to reach $37^{\circ} \mathrm{C}$ at the trachea. The MR850 $\mathrm{HH}$ maintains the temperature along the inspiratory limb by monitoring the temperature at the Y-piece and controlling the power delivered to the heater wire. In this proto$\mathrm{col}$, the $\mathrm{HH}$ was set in the manual mode to deactivate the automatic mode and to maintain the target temperature of $40^{\circ} \mathrm{C}$ at the Y-piece and $37^{\circ} \mathrm{C}$ at the chamber outlet.

$\mathrm{AH}$ was measured with the psychrometric method. ${ }^{11} \mathrm{~A}$ psychrometric device consists of 2 temperature probes. The surface of one probe is covered with water and is termed the wet probe; the second probe is exposed to air and is termed the dry probe. As a result of evaporation of water from the wet probe, the temperature measured by the wet thermometer is lower than that measured by the dry. The difference in the temperatures measured with these thermic probes yields the RH from Mollier's psychrometric diagram. ${ }^{11}$ The $\mathrm{AH}\left(\mathrm{mg} \mathrm{H}_{2} \mathrm{O} / \mathrm{L}\right)$ is determined with the equation $\left(\mathrm{AH}_{\mathrm{S}} \times \mathrm{RH}\right) / 100$, which is computed with the temperature measured with the dry probe:

$$
\mathrm{AH}_{\mathrm{S}}\left(\mathrm{mg} \mathrm{H} \mathrm{H}_{2} \mathrm{O} / \mathrm{L}\right)=16.451563-(0.731 \times \mathrm{T})+
$$

$\left(0.03987 \times \mathrm{T}^{2}\right)$ for a dry temperature between

$$
24.1^{\circ} \mathrm{C} \text { and } 38^{\circ} \mathrm{C}
$$

or

$$
\begin{array}{r}
\mathrm{AH}_{\mathrm{S}}\left(\mathrm{mg} \mathrm{H}_{2} \mathrm{O} / \mathrm{L}\right)=6.0741+(0.1039 \times \mathrm{T})+ \\
\left(0.02266 \times \mathrm{T}^{2}\right) \text { for a dry temperature between }
\end{array}
$$

$10^{\circ} \mathrm{C}$ and $24.1^{\circ} \mathrm{C}$, where $\mathrm{T}$ is the measured dry temperature expressed in ${ }^{\circ} \mathrm{C}$.

According to the manufacturers, the accuracy of each probe is $\pm 0.1^{\circ} \mathrm{C}$ and $\pm 0.04 \mathrm{ohms}$ at $0^{\circ} \mathrm{C}$, and the tolerance is $\pm 0.333 \times(0.3+0.005 \times$ temperature $)$ or $100 \pm 0.10 \mathrm{ohms}$ at $0^{\circ} \mathrm{C}$. Therefore, the accuracy is $0.2^{\circ} \mathrm{C}$ for the temperature difference with each probe. With this accuracy, the error in estimating the relative humidity at $20^{\circ} \mathrm{C}$ is $1.3 \%, 1.4 \%$, and $1.9 \%$ for $10 \%, 50 \%$, and $100 \%$ relative humidity, respectively.

The 2 thermal probes were positioned in the inspiratory part of a double circuit that separated the inspiratory and expiratory gas flows through unidirectional valves (Fig. $1)$. Then the room temperature was measured and its value was used for temperature probe calibration. Each temperature probe was calibrated at 2 temperature points (ie, ambient temperature and iced water assumed to be at $0^{\circ} \mathrm{C}$ ) and plugged into the data logger, as were pressure and flow signals after calibration. Then one of the probes was covered with wet cotton.

Each ventilator was fully checked with the $\mathrm{HH}$ in place and switched off, according to the procedure described in the manufacturer's user manual. This included the detection of leaks and determination of breathing circuit compliance. If available, the algorithm for leak compensation was switched off to unmask the presence of a leak. A pneumotachometer was inserted at the ventilator outlet to define the breathing cycle. The psychrometric device was fitted between the Y-piece and the test lung, and the port for pressure was inserted between the psychrometric device and the test lung (Fig. 1). We inserted a pneumotachometer at the ventilator outlet to have the same set up in both protocols, but we didn't insert a pneumotachometer at the Y-piece as in the first protocol because we were concerned with a potential problem in the quality of flow measurement due to humidity. However, because the G5 ventilator uses its own pneumotachometer for proper functioning, with this ventilator we kept the pneumotachometer between the psychrometric device and the pressure port. We took great care to avoid any leak into the experimental set-up. We verified this by checking the airway pressure stability during an end-inspiratory pause with the complete experimental set-up in place.

We then switched on the HH, set in manual compensation and invasive mode. The following ventilator settings were applied for $45 \mathrm{~min}$ to reach temperature equilibrium within the system (breathing circuit, measurement set-up, and lung model), according to the manufacturer's recommendation (Fisher-Paykel) ${ }^{11}: \mathrm{V}_{\mathrm{T}}=500 \mathrm{~mL}$, volume controlled mode with a constant inspiratory flow of $60 \mathrm{~L} / \mathrm{min}$, $\mathrm{F}_{\mathrm{IO}_{2}}=0.21, \mathrm{PEEP}=12 \mathrm{~cm} \mathrm{H}_{2} \mathrm{O}$, inspiratory pause $=0.2 \mathrm{~s}$, and $\mathrm{f}=30$ breaths $/ \mathrm{min}$. This value for $\mathrm{f}$ was used first in each test because the $\mathrm{HH}$ better performs at higher minute ventilation. Then $\mathrm{V}_{\mathrm{T}}$ was reduced to $100 \mathrm{~mL}$ and inspira- 
tory flow to $20 \mathrm{~L} / \mathrm{min}$, with the other settings being unchanged, and we started continuous acquisition of the flow, pressure, and temperature signals at $200 \mathrm{~Hz}$.

Once plateaus in both temperatures were reached, as defined by a change in temperature of $1 \%$ or less (see supplementary materials at http://www.rcjournal.com), $\mathrm{V}_{\mathrm{T}}$ was changed by $20 \mathrm{~mL}$. For each set $\mathrm{V}_{\mathrm{T}}$, once a new temperature plateau, as defined previously, was reached, 10 breaths were counted, and then the next $\mathrm{V}_{\mathrm{T}}$ was applied. This continued up to $\mathrm{V}_{\mathrm{T}}=280 \mathrm{~mL}$. Then $\mathrm{f}$ was reduced to 15 breaths/min, and $\mathrm{V}_{\mathrm{T}}$ was set back to $100 \mathrm{~mL}$ under continuous monitoring of temperature. Once the temperature leveled off, we repeated the stepwise increments of $\mathrm{V}_{\mathrm{T}}$ up to $280 \mathrm{~mL}$.

With the neonate breathing circuit, this procedure was repeated up to and including the activation of the $\mathrm{HH}$, set in manual compensation and invasive mode. The following ventilator settings were applied for $45 \mathrm{~min}$ to reach temperature equilibrium within the system (breathing circuit, measurement set-up, and lung model), according to the manufacturer's recommendation (Fisher-Paykel) and previous works by others ${ }^{11}: \mathrm{V}_{\mathrm{T}}=300 \mathrm{~mL}$, volume controlled mode with a constant inspiratory flow of $20 \mathrm{~L} / \mathrm{min}$, $\mathrm{F}_{\mathrm{IO}_{2}}=0.21, \mathrm{PEEP}=12 \mathrm{~cm} \mathrm{H}_{2} \mathrm{O}$, inspiratory pause $=0.2 \mathrm{~s}$, $\mathrm{f}=30$ breaths $/ \mathrm{min}$. This value $\mathrm{f}$ was used first in each test because the $\mathrm{HH}$ performs better at higher minute ventilation. Then $\mathrm{V}_{\mathrm{T}}$ was reduced to $100 \mathrm{~mL}$, with the other settings being unchanged, and we initiated continuous acquisition of flow, pressure, and temperature signals at $200 \mathrm{~Hz}$.

Once both temperatures leveled off, as defined by a change in temperature of $1 \%$ or less, $V_{T}$ was changed by $20 \mathrm{~mL}$. For each set $\mathrm{V}_{\mathrm{T}}$, once a new temperature plateau, as defined previously, was reached, 10 breaths were counted, and then the next $\mathrm{V}_{\mathrm{T}}$ was applied. This continued up to $\mathrm{V}_{\mathrm{T}}=280 \mathrm{~mL}$. Then $\mathrm{f}$ was reduced to 15 breaths/min, and $\mathrm{V}_{\mathrm{T}}$ was set back to $100 \mathrm{~mL}$ under continuous monitoring of temperature. Once the temperature leveled off, we repeated the stepwise increments of $\mathrm{V}_{\mathrm{T}}$ up to $280 \mathrm{~mL}$.

During every condition, we watched the $\mathrm{HH}$ alarm and checked for temperature at both the chamber outlet and the Y-piece to ensure the targets were maintained.

\section{Data Analysis}

For the first protocol, inspired $\mathrm{V}_{\mathrm{T}}$ was obtained by numerical integration of the flow signal. $\mathrm{V}_{\mathrm{T}}$ was measured over the last 10 breaths for each nominal set $\mathrm{V}_{\mathrm{T}}$ in each condition at the Y-piece with Acqknowledge 4.4 software (Biopac Systems). For proper comparison with the ventilator indication (BTPS), all volumes inferred via the pneumotachometer (ie, in atmospheric temperature and pressure condition) were normalized in BTPS condition as follows:

$$
\begin{aligned}
& \mathrm{V}_{\mathrm{T}}(\mathrm{BTPS})=\mathrm{V}_{\mathrm{T}}\left(\mathrm{AT} \times \mathrm{P}_{\mathrm{A}} \times \mathrm{RH}\right) \times(310.15 / \mathrm{T}) \times \\
& \left\{\left[\mathrm{P}_{\mathrm{A}}-\mathrm{RH} \times \mathrm{P}_{\mathrm{sat}}(\mathrm{T})\right] /\left[\left(\mathrm{P}_{\mathrm{A}}+\mathrm{PEEP}\right)-\mathrm{P}_{\mathrm{sat}}(310.15)\right]\right\}
\end{aligned}
$$

where AT is the ambient temperature expressed in ${ }^{\circ} \mathrm{K}, \mathrm{P}_{\mathrm{A}}$ is the ambient air pressure, $\mathrm{RH}$ is the relative humidity of the ambient air, $\mathrm{P}_{\text {sat }}$ is the saturation pressure of water (or equilibrium vapor pressure) and $\mathrm{T}$ defined above.

For the second protocol, $\mathrm{AH}$ was averaged during the insufflation phase of the breathing cycle over each of the last 10 breaths in each condition. Because ICU ventilators adapt differently when $\mathrm{V}_{\mathrm{T}}$ is changed (see the supplementary materials at http://www.rcjournal.com), we measured the mean inspiratory flow and the inspiratory time from the pneumotachometer at the ventilator outlet.

\section{Statistical Analysis}

Values are shown as median (first-third quartiles) unless otherwise stated. For the first protocol, a linear regression model was performed for each circuit/f combination with $\mathrm{V}_{\mathrm{T}}$ measured at the $\mathrm{Y}$-piece as the dependent variable and set $\mathrm{V}_{\mathrm{T}}$ (used as a continuous variable) and ventilator with their interaction as the explanatory variables. For a given condition, the mean \pm SD intercept and slope were estimated for each ventilator. The relative error in set $V_{T}$ was computed as (set $\mathrm{V}_{\mathrm{T}}$ - measured $\mathrm{V}_{\mathrm{T}}$ )/set $\mathrm{V}_{\mathrm{T}}$ over the whole range of $\mathrm{V}_{\mathrm{T}}$, expressed as percentage set $\mathrm{V}_{\mathrm{T}}$ and compared across ventilators with a Kruskal-Wallis test with pairwise post hoc analysis when appropriate. The commonly used $10 \%$ boundaries in the relative error defined the limits of accuracy of $\mathrm{V}_{\mathrm{T}}$ delivery.

For the second protocol, a linear regression model was performed for each $\mathrm{f} /$ circuit combination with $\mathrm{AH}$ as the dependent variable and set $\mathrm{V}_{\mathrm{T}}$ (used as a continuous variable) and ventilator with their interaction as the explanatory variables. We used the results of this analysis to compute the minimal $\mathrm{V}_{\mathrm{T}}$ that, for each ventilator and $\mathrm{f} /$ circuit combination, fell inside the upper $95 \% \mathrm{CI}$ of the $33 \mathrm{mg} / \mathrm{L}$ AH target.

$P$ values $<.05$ indicated statistical significance. Statistical analysis was performed using the R software, version 3.2 (R Foundation for Statistical Computing, Vienna, Austria).

\section{Results}

\section{Protocol 1}

For each ventilator, $400 \mathrm{~V}_{\mathrm{T}}$ values (ie, 10 nominal set $V_{T}$ values $\times 10$ breaths $\times 2$ breathing circuits $\times 2 \mathrm{f}$ ) were generated and split into $4 \mathrm{f} / \mathrm{breathing}$ circuit combinations of 100 measurements each. The average slope $(95 \%$ CI) for the 5 ventilators between measured $V_{T}$ versus set $V_{T}$ 
was 0.93 (0.92-0.93), 0.93 (0.93-0.94), 0.91 (0.90-0.91), and $0.91(0.90-0.91) \mathrm{mL} / \mathrm{mL}$ for $\mathrm{f} 15$ and $\mathrm{f} 30$ adult and neonate circuits, respectively $(P<.05$ vs 1 in each instance), indicating a systematic under-delivery of $\mathrm{V}_{\mathrm{T}}$ (Table 1). For each ventilator, a positive slope deviation means that the underestimation of set $\mathrm{V}_{\mathrm{T}}$ is less than the mean (Table 1), with the opposite being true for a negative slope. For example, for the f15/adult circuit combination, Carestation, G5, and Servo-U performed better than PB980 and V500. Across the f/breathing circuit combinations, V500 had consistent negative slope deviations and Servo-U had consistent positive slope deviations, whereas the slope deviation sign with the other 3 ventilators changed depending on the condition. The under-delivery of $\mathrm{V}_{\mathrm{T}}$ was greater with neonate circuits than with adult circuits. As shown in Figure 2, with the adult circuits at both $\mathrm{f}$ settings, the relationship of measured $\mathrm{V}_{\mathrm{T}}$ to set $\mathrm{V}_{\mathrm{T}}$ fell along the identity line for Carestation and Servo-U ventilators. The relationship was below the identity line for the other 3 ventilators with the adult circuit and for each ventilator with the neonate circuits (Fig. 2). The relative error in set $\mathrm{V}_{\mathrm{T}}$ over the whole $\mathrm{V}_{\mathrm{T}}$ range is shown in Figure 3 with the $\pm 10 \%$ boundary for accuracy. Only the Carestation and Servo-U ventilators for $\mathrm{f} 15$ and $\mathrm{f} 30$ /adult combinations delivered $\mathrm{V}_{\mathrm{T}}$ within the $10 \%$ range of accuracy (Fig. 3).

\section{Protocol 2}

Overall, $\mathrm{AH}$ increased linearly with increasing $\mathrm{V}_{\mathrm{T}}$ (Fig. 4). The results of the linear regression models of $\mathrm{AH}$ versus set $\mathrm{V}_{\mathrm{T}}$ are given for each $\mathrm{f} /$ circuit combination (see supplementary materials at http://www.rcjournal.com). The models were used to compute the minimal volume at which the recommended $33 \mathrm{mg} / \mathrm{L} \mathrm{AH}$ was reached. This volume differed across ventilators and could not be reached with some ventilators in some $\mathrm{f} / \mathrm{breathing}$ circuit combinations (Table 2). The single situation in which a minimal volume accommodating $33 \mathrm{mg} / \mathrm{L} \mathrm{AH}$ was reached for all the ventilators was $\mathrm{f} 30$ with an adult breathing circuit (Table 2). In this combination, the minimal volumes were 100, 150, 190, 160, and $100 \mathrm{~mL}$ for G5, Carestation, PB980, Servo-U, and V500, respectively (Table 2). No relationship was found between $\mathrm{AH}$ and inspiratory pattern (ie, inspiratory time and flow) (data not shown).

\section{Discussion}

The main findings of this bench study performed over a low $\mathrm{V}_{\mathrm{T}}$ range were that, in most instances, ICU ventilators under-delivered $\mathrm{V}_{\mathrm{T}}$, and that the recommended $\mathrm{AH}$ target was attained in these ventilators only if the $\mathrm{f} 30$ and adult breathing circuit combination is used.

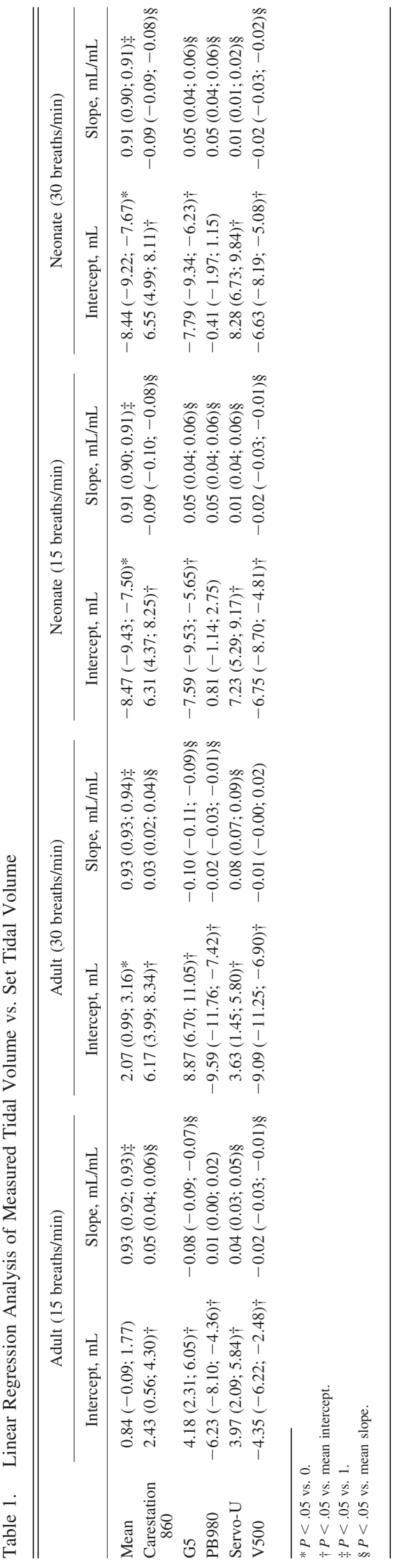



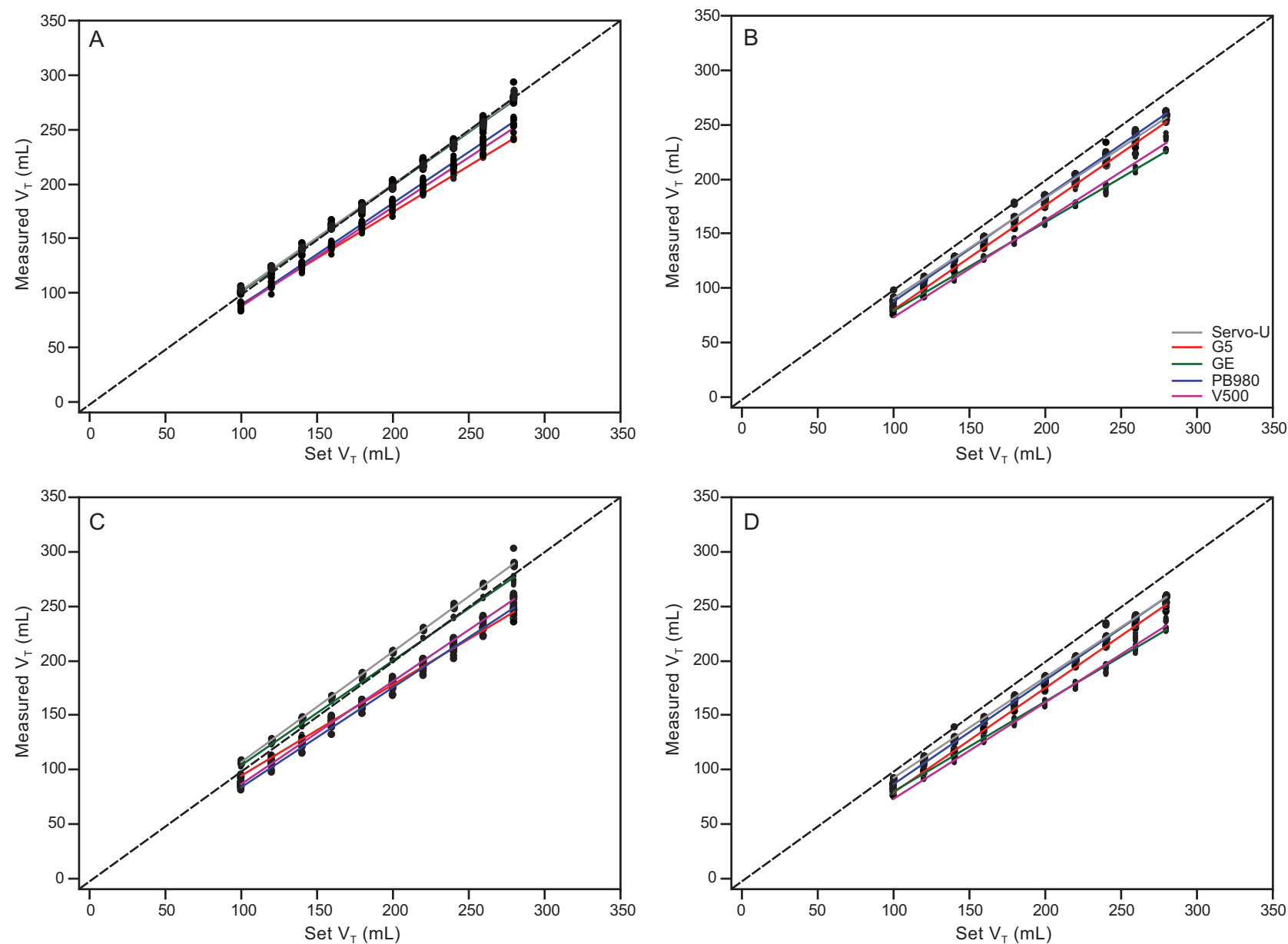

Fig. 2. Relationships of measured tidal volume $\left(V_{T}\right)$ at the $Y$ piece to set $V_{T}$ across ventilators, for adult or neonate circuits at breathing frequency $(f)$ of 15 breaths $/ \mathrm{min}$ or 30 breaths/min. Every measured $V_{T}$ is plotted. The identity lines and the regression lines are shown. (A) Adult circuit with 15 breaths/min. (B) Neonatal circuit with 15 breaths/min. (C) Adult circuit with 30 breaths/min. (D) Neonatal circuit with 30 breaths/min. BTPS = body temperature and pressure saturated.

\section{Accuracy of $\mathbf{V}_{T}$ Delivery}

The present low $\mathrm{V}_{\mathrm{T}}$ range has not previously been systematically investigated in vitro. We found that the mean effect of the five ICU ventilators was a 7\% under delivery of $\mathrm{V}_{\mathrm{T}}$ with differences across ventilators and f/circuit combinations. In a comprehensive bench study Garnier et al ${ }^{12}$ tested the accuracy of delivering 200,300, 400, and $800 \mathrm{~mL}$ $\mathrm{V}_{\mathrm{T}} \mathrm{S}$ in volume controlled continuous mandatory ventilation mode with 13 ICU ventilators. Using the same ICU ventilators as in our study, these authors found that, over the whole range of $\mathrm{V}_{\mathrm{T}}$ values, accuracy fell into the $\pm 10 \%$ range except for 1 ventilator. These results vary from our study, in which only 2 out of 5 recent ventilator models fell into the $\pm 10 \%$ accuracy.

Our results for target $\mathrm{V}_{\mathrm{T}}$ were not influenced by gas compression within the circuit because we carefully measured circuit compliance and entered these values into the ventilator prior to the experiment.
The adaptation of ventilator to $\mathrm{V}_{\mathrm{T}}$ change differed among the ventilators. Some increased inspiratory time others increased inspiratory flow. These adaptations are not likely to explain the discrepancy between ventilators regarding VT accuracy because the Carestation and Servo-U ventilators, with better accuracy using the adult circuit at both fs, used opposite strategies when facing acute VT changes (see the supplementary materials at http://www.rcjournal. com).

The kind of circuit played a role in the under-delivery of $\mathrm{V}_{\mathrm{T}}$. The neonate circuit as compared to the adult circuit at both $\mathrm{f}$ was associated with under-delivery of $\mathrm{V}_{\mathrm{T}}$ because every ICU ventilator tested fell outside the $\pm 10 \%$ boundary of delivery accuracy. This result can be due to either the neonate circuit per se or the setting of the ventilator in the neonate category. Our study did not determine differences between each component. Jaecklin et $\mathrm{al}^{13}$ bench tested 6 neonatal ventilators set in pressure control with adaptive targeting mode. When the lung model was set for full-term 

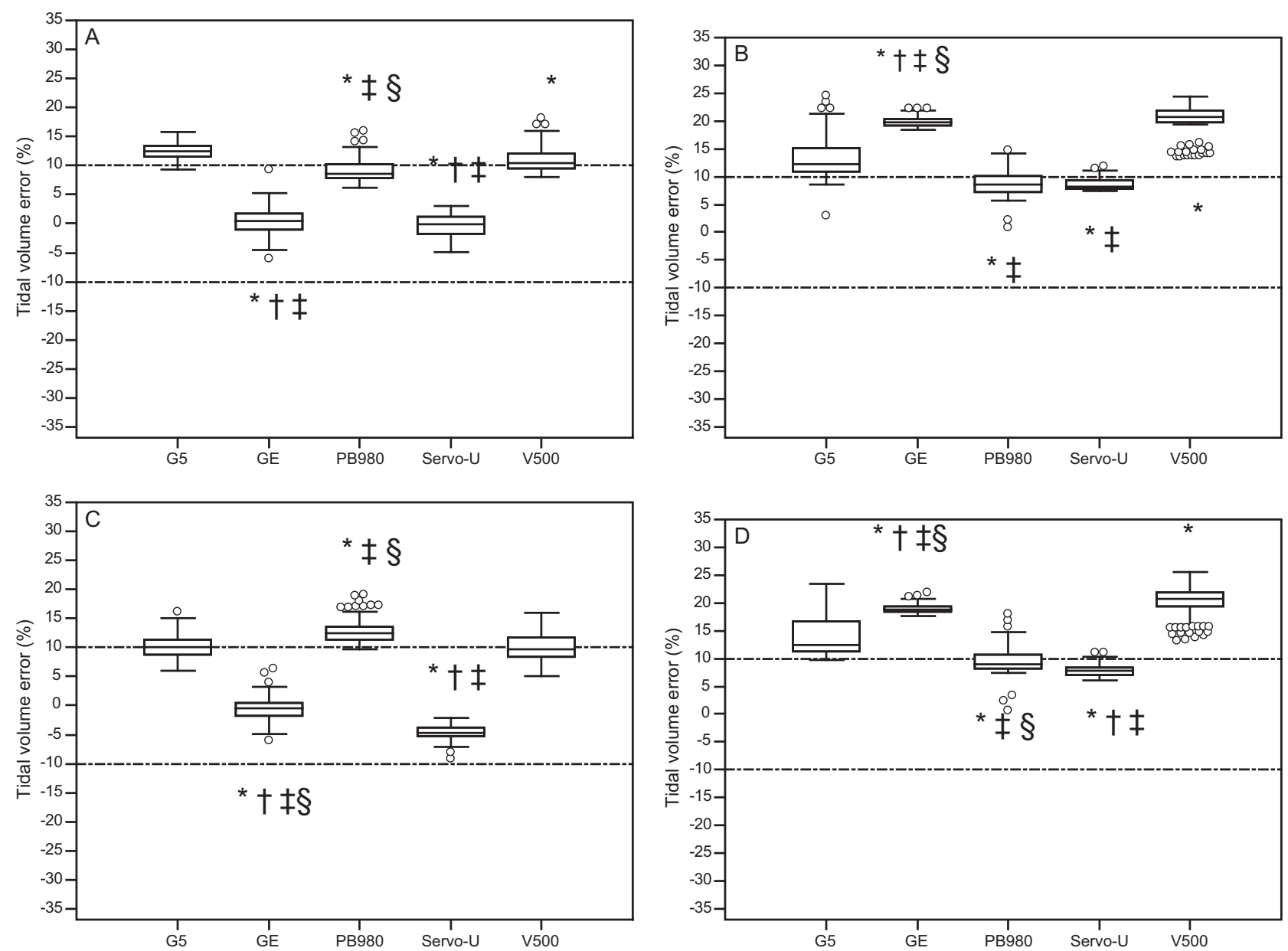

Fig. 3. Box-and-whisker plots of the relative error for tidal volume $\left(V_{T}\right)$ across ventilators for each circuit and breathing frequency ( $f$ ) combination. (A) Adult circuit with at 15 breaths/min (B) Neonatal circuit at 15 breaths $/ \mathrm{min}$. (C) Adult circuit at 30 breaths/min. (D) Neonatal circuit at 30 breaths $/ \mathrm{min}$. The relative error was computed as the ratio of the difference between set and measured $V_{T}$ to set $V_{T}$. For each ventilator, the distribution of the 100 measurements of $V_{T}$ over the whole range of $V_{T}$ is used. Symbols indicate post-hoc analysis of the Kruskal-Wallis test with pairwise differences between ventilators for $P<.05$ vs. * G5, † PB980, $\ddagger$ V500, and $\S$ Servo-U.

neonate settings (ie, lung compliance $=4 \mathrm{~mL} / \mathrm{cm} \mathrm{H}_{2} \mathrm{O}$ and resistance $\left.50 \mathrm{~cm} \mathrm{H}_{2} \mathrm{O} / \mathrm{L} / \mathrm{s}\right)$, the $25-\mathrm{mL}$ set $\mathrm{V}_{\mathrm{T}}$ was delivered with an error range of $76-134 \%$ across the ventilators. When the lung model was set for preterm neonate settings (ie, lung compliance $=2 \mathrm{~mL} / \mathrm{cm} \mathrm{H}_{2} \mathrm{O}$ and resistance $50 \mathrm{~cm} \mathrm{H}_{2} \mathrm{O} / \mathrm{L} / \mathrm{s}$ ), the $8-\mathrm{mL}$ set $\mathrm{V}_{\mathrm{T}}$ was delivered with an error range of $68-147 \%$. In each condition, 2 ventilators fell outside the $\pm 10 \%$ accuracy range. Similar to our results, the study by Jaecklin et al, ${ }^{13}$ in which 3 ventilators were of the same brand as in our study (ie, Evita XL and V500, Galileo and G5, Servo-i and Servo-U), suggests that recent ICU ventilators do not perform better than previous neonate-dedicated ICU ventilators regarding accuracy of $\mathrm{V}_{\mathrm{T}}$ delivery.

\section{AH Recommended Threshold}

We assessed AH using the psychrometric method, which required thorough and careful attention to obtain reliable results. Hence a comparison of our results with previous bench studies, in particular that by Lellouche et $\mathrm{al}^{11}$ is warranted. We used a 45-min period to reach thermal equilibrium prior to obtaining measurements, whereas Lellouche et al ${ }^{11}$ waited $3 \mathrm{~h}$. We used this strategy because we continuously monitored the temperature at the Y-piece and found that equilibrium was reached by this time point. Continuous monitoring of temperature during the acute changes in $\mathrm{V}_{\mathrm{T}}$ in our second protocol allowed us to move our $\mathrm{V}_{\mathrm{T}}$ steps forward. We set the $\mathrm{HH}$ in manual compensation mode to deactivate the automatic mode. At the end of our experiment (ie, at $\mathrm{V}_{\mathrm{T}}=280 \mathrm{~mL}$ ), AH exhibited the highest value as expected, which amounted to 30-34 $\mathrm{mg} \mathrm{H}_{2} \mathrm{O} / \mathrm{L}$ for f15/adult, 29-34 $\mathrm{mg} \mathrm{H}_{2} \mathrm{O} / \mathrm{L}$ for f15/neonat, 34-37 $\mathrm{mg} \mathrm{H}_{2} \mathrm{O} / \mathrm{L}$ for f30/adult, and 30-34 $\mathrm{mg} \mathrm{H}_{2} \mathrm{O} / \mathrm{L}$ for f30/neonate, respectively. These values are slightly lower than those found by Lellouche et $\mathrm{al}^{11}$ when they tested normal ambient air temperature $\left(22-24^{\circ} \mathrm{C}\right)$ using either the 

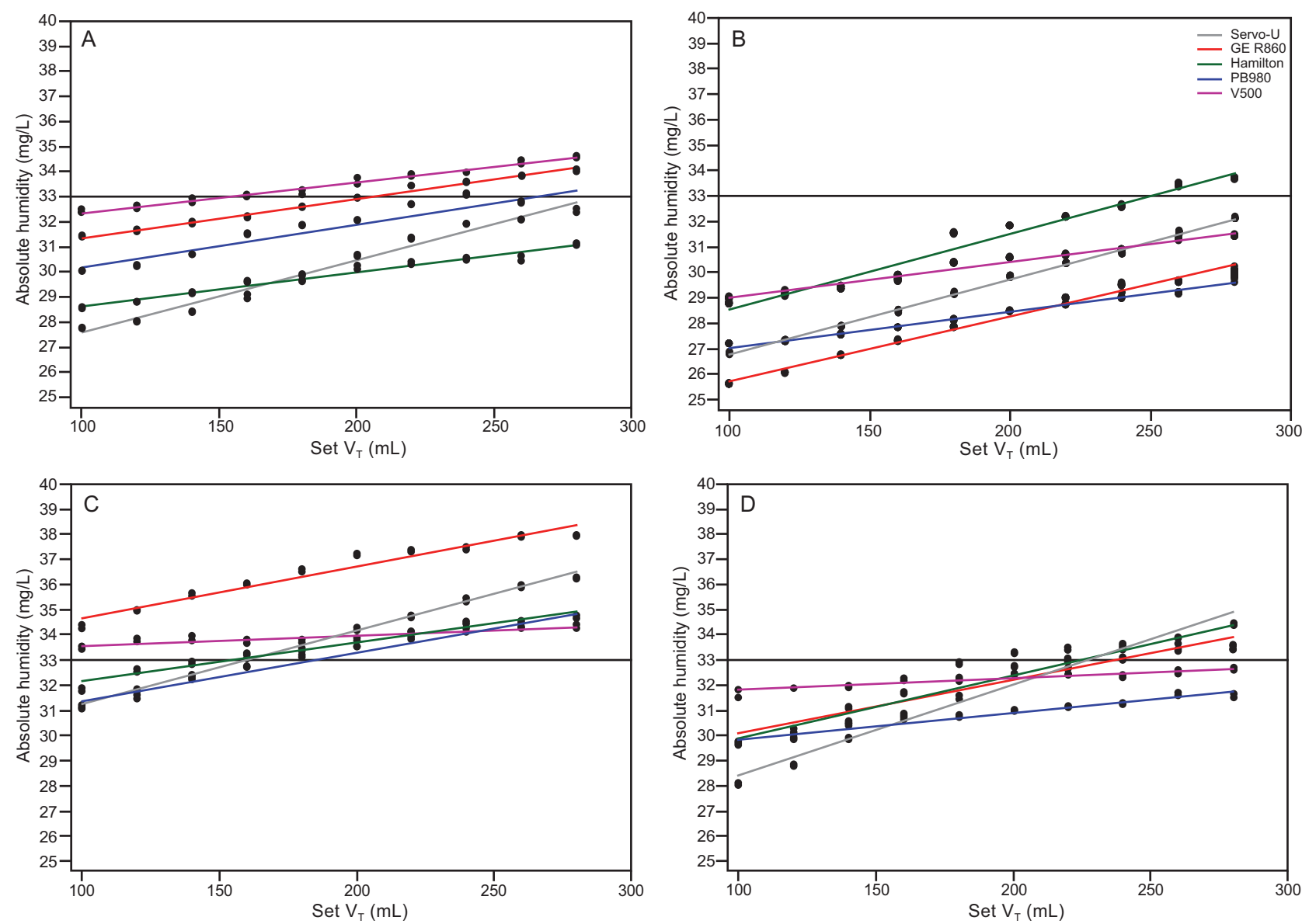

Fig. 4. Relationships of absolute humidity to set tidal volume (VT) across ventilators, for adult or neonate circuits at 15 or 30 breaths/min breathing frequency (f). A: Adult circuit with at 15 breaths/min, B: neonatal circuit at 15 breaths/min, C: adult circuit at 30 breaths/min, and $\mathrm{D}$ : neonatal circuit at 30 breaths $/ \mathrm{min}$. Every measurement of absolute humidity is plotted. Horizontal lines show $33 \mathrm{mg} / \mathrm{L}$ absolute humidity, which is the target for optimal humidity in ICU patients receiving mechanical ventilation.

Table 2. Minimal Volume to Meet the Recommended Absolute Humidity Target*

\begin{tabular}{lcccc}
\hline \hline & $\begin{array}{c}\text { Adult (15 } \\
\text { breaths/min) }\end{array}$ & $\begin{array}{c}\text { Adult (30 } \\
\text { breaths/min) }\end{array}$ & $\begin{array}{c}\text { Neonate (15 } \\
\text { breaths/min) }\end{array}$ & $\begin{array}{c}\text { Neonate (30 } \\
\text { breaths/min) }\end{array}$ \\
\hline GE & 225 & 100 & None & 250 \\
G5 & None & 150 & 250 & 230 \\
PB980 & 260 & 190 & None & None \\
Servo-U & None & 160 & None & None \\
V500 & 150 & 100 & None & None \\
& & & & \\
* The recommended absolute humidity target was 33 mg/L. & \\
\hline
\end{tabular}

automatic compensation setting of the $\mathrm{HH}$ or $\mathrm{HH}$ chamber-to-Y-piece temperature gradient of $37-40^{\circ} \mathrm{C}$, which ranged roughly from $31-36^{\circ} \mathrm{C}$. This difference is likely explained primarily by the minute ventilation, which was a pooled value between 10 and $20 \mathrm{~L} / \mathrm{min}$ in the study by Lellouche et al, ${ }^{11}$ whereas the highest minute ventilation in our study was $8.4 \mathrm{~L} / \mathrm{min}$. Other reasons for the differences are between-bench study variability and the loss of water vapor due to condensation, which occurs between the end of the inspiratory limb and entry into the lung model. We observed some condensation upstream the location of the psychrometric device, but we did not quantify it.

Inspiratory times ranged from 0.3 to $0.84 \mathrm{~s}$ during our experiment, which seemed short for adult ventilation. In severe ARDS with very low compliance and short time constants of the respiratory system, it is common to set $\mathrm{f}$ at the ventilator to 35 breaths/min, which results in a total breath length of $1.71 \mathrm{~s}$. For a 1:1 or 1:2 inspiratory-expiratory ratio, the corresponding inspiratory times are $0.85 \mathrm{~s}$ and $0.53 \mathrm{~s}$, which are near the range of inspiratory times we used.

We found that the $\mathrm{V}_{\mathrm{T}}$ course of $\mathrm{AH}$ significantly differed across ventilators. There are several explanations for this finding. One could be the bias flow in the ventilator; ie, the higher the bias flow, the higher the flow passing through the $\mathrm{HH}$ and hence the higher the work to be done by the $\mathrm{HH}$ to reach the temperature targets. We used the 
ventilator default setting for the bias flow in our experiment: $10 \mathrm{~L} / \mathrm{min}$ for $\mathrm{G} 5,10 \mathrm{~L} / \mathrm{min}$ for the Carestation 860 , $10 \mathrm{~L} / \mathrm{min}$ for the PB980, $2 \mathrm{~L} / \mathrm{min}$ for the Servo-U, and $2 \mathrm{~L} / \mathrm{min}$ for the V500. The 2 ventilators with the lowest bias flow did not perform better than the other 3 ventilators with regard to achieving the $\mathrm{AH}$ target. The lack of significant impact of bias flow could result from better functioning of the $\mathrm{HH}$ in the presence of the bias flow, which makes the flow across the chamber steadier. We expected that the way the ventilator adapts to acute $V_{T}$ changes may contribute to variations in $\mathrm{AH}$. However, there was no difference between ventilators with flow or with inspiratory time adjustment during $\mathrm{V}_{\mathrm{T}}$ change and $\mathrm{AH}$.

During this experiment, we did not observe any temperature alarm at either the chamber or the Y-piece, indicating adequate $\mathrm{HH}$ function. Of particular note, we did not observe any low-temperature alarm, which would have provided information regarding insufficient humidification of the inspired air. In the MR850 device, the low-temperature alarm is based on the lowest temperature value measured between the chamber and outlet and the Y-piece, which is an estimate of the dew point. The warning threshold of $35.4^{\circ} \mathrm{C}$ is activated first with a light, then with a light and a warning, depending on the time elapsed and the extent of departure below the threshold. Therefore, our findings resulted in an appropriate choice of $\mathrm{f}$ and circuit combination in this setting of low $\mathrm{V}_{\mathrm{T}}$ that allowed efficient $\mathrm{AH}$ above the threshold of the low-temperature alarm in the $\mathrm{HH}$.

The fact that $\mathrm{AH}$ is better with the f30/adult circuit combination is in agreement with the physics theory. First, it is intuitive that the faster the transit time of the gas flowing through a structure, the lower the time allowed for gas cooling, condensing, and losing humidity, even though the gas is warmed downstream. This consideration favors using $\mathrm{f} 30$ rather than $\mathrm{f} 15$. Second, the area for thermal exchange with the environment is given by $l \times 2 \pi r$, where $l$ is the length and $r$ is the radius of the structure subjected to thermal exchange. The mass of gas being proportional to $l \times \pi r^{2}$, the ratio of the exchange surface area to the gas mass not to cool is $2 / r$. The lower $r$ and hence the higher the ratio $2 / r$, the greater the exchange surface area relative to the gas mass and the greater the likelihood for this gas mass to cool (and thus to condensate and reduce $\mathrm{AH}$ ). Therefore, the adult circuit is better suited. From the above considerations, and given our results regarding the capability of the whole system to maintain $\mathrm{AH}$ at or above $33 \mathrm{mg} / \mathrm{L}$, it follows that it would be more efficient to design an $\mathrm{HH}$ to reach $43^{\circ} \mathrm{C} 100 \% \mathrm{RH}$ in the chamber, allowing condensation into the circuit, to achieve $40^{\circ} \mathrm{C}$ $100 \% \mathrm{RH}$ at the $\mathrm{Y}$-piece and hence $37^{\circ} \mathrm{C} 100 \% \mathrm{RH}$ at the trachea.

During invasive mechanical ventilation, an alternative to an $\mathrm{HH}$ is a heat-and-moisture exchanger (HME). Both devices perform similarly for efficiently conditioning in- spired air. ${ }^{14}$ However, use of an HME is not recommended during mechanical ventilation for ARDS because of the instrumental dead space it adds to the physiologic dead space. This is particularly relevant in the conditions of our study, in which very low $\mathrm{V}_{\mathrm{T}} \mathrm{s}$ were used. This is the main reason why HMEs were not tested in this study.

\section{Limitations and Strengths}

Our study is the first to systematically assess the role of a large range of low $\mathrm{V}_{\mathrm{T}}$ with different breathing circuits on $\mathrm{AH}$. This is a bench study and these results cannot be extrapolated to patients. As previously mentioned, we did not quantify the condensation and hence the loss of water between the Y-piece and lung model entry. We did not measure condensation in our experiment because the condensation in the circuit (from chamber outlet to Y-piece) is the amount of humidity that does not reach the respiratory tract of the patient. This is a component of the potential lack of efficiency of the heater. The condensation in this part of the set-up in our experiment would not have modified AH measurement. The condensation that happened downstream from the Y-piece (ie, in the inspiratory limb of the psychrometric device) was directly due to the set up and would not have happened in the clinical setting. As a result, our measurement underestimated $\mathrm{AH}$ compared to what would be observed in the clinical setting. However, it is worth noting that, during mechanical ventilation with $\mathrm{HH}$, condensation occurs in the catheter mount located between the Y-piece and proximal tip of the endotracheal tube. Compared to our bench study, in patients the water loss resulting from condensation should be lower because the internal volume of the catheter mount is lower than the devices between the Y-piece and lung model inlet, and because warm air expelled from the patient prevents condensation. Condensation downstream of the thermic probes, if there is any, including the expiratory line, has no impact on our measurements of $\mathrm{AH}$.

\section{Clinical Implications}

The main implication of our results is that they inform clinicians of the need to select an adequate ICU ventilator, breathing circuit, and $\mathrm{f}$ when considering ECMO for a patient with ARDS. The results of our study can help clinicians gauge the kind of ventilator, breathing circuit, and $\mathrm{f}$ depending on the size of the set $\mathrm{V}_{\mathrm{T}}$. Our results indicate that an adult circuit and $\mathrm{f}=30$ breaths $/ \mathrm{min}$ should be selected for patients with ARDS who are receiving ECMO. The next step is to address these issues in a clinical study that replicates the bench conditions of our experiment. 


\section{Absolute Humidity and Delivery of Low $V_{T}$}

\section{Conclusion}

Low values of $\mathrm{V}_{\mathrm{T}}$ were under-delivered via the tested ICU ventilators by an average of 7-9\%. The recommended target $\mathrm{AH}$ of $33 \mathrm{mg} / \mathrm{L}$ could be reached with every ventilator only with the use of the combination of an adult circuit and a breathing frequency of 30 breaths/min.

\section{REFERENCES}

1. Combes A, Brodie D, Bartlett R, Brochard L, Brower R, Conrad S, et al. Position paper for the organization of extracorporeal membrane oxygenation programs for acute respiratory failure in adult patients. Am J Respir Crit Care Med 2014;190(5):488-496.

2. Serpa Neto A, Schmidt M, Azevedo LC, Bein T, Brochard L, Beutel $\mathrm{G}$, et al. Associations between ventilator settings during extracorporeal membrane oxygenation for refractory hypoxemia and outcome in patients with acute respiratory distress syndrome: a pooled individual patient data analysis: mechanical ventilation during ECMO. Intensive Care Med 2014;42(11):1672-1684.

3. Schmidt M, Pellegrino V, Combes A, Scheinkestel C, Cooper DJ, Hodgson C. Mechanical ventilation during extracorporeal membrane oxygenation. Crit Care 2014;18(1):203-213.

4. Schmidt M, Stewart C, Bailey M, Nieszkowska A, Kelly J, Murphy $\mathrm{L}$, et al. Mechanical ventilation management during extracorporeal membrane oxygenation for acute respiratory distress syndrome: a retrospective international multicenter study. Crit Care Med 2015; 43(3):654-664.

5. Pelosi P, Chiumello D, Severgnini P, De Grandis CE, Landi L, Chierichetti LM, et al. Performance of heated wire humidifiers: an in vitro study. J Crit Care 2007;22(3):258-264.
6. American Association for Respiratory Care, Restrepo RD, Walsh BK. Humidification during invasive and noninvasive mechanical ventilation: 2012. Respir Care 2012;57(5):782-788

7. Lyazidi A, Thille AW, Carteaux G, Galia F, Brochard L, Richard JC. Bench test evaluation of volume delivered by modern ICU ventilators during volume-controlled ventilation. Intensive Care Med 2010; 36(12):2074-2080.

8. Mauri T, Bellani G, Grasselli G, Confalonieri A, Rona R, Patroniti $\mathrm{N}$, et al. Patient-ventilator interaction in ARDS patients with extremely low compliance undergoing ECMO: a novel approach based on diaphragm electrical activity. Intensive Care Med 2013;39(2): 282-291.

9. Brunet F, Mira JP, Belghith M, Monchi M, Renaud B, Fierobe L, et al. Extracorporeal carbon dioxide removal technique improves oxygenation without causing overinflation. Am J Respir Crit Care Med 1994;149(6):1557-1562.

10. Bellani G, Laffey JG, Pham T, Fan E, Brochard L, Esteban A, et al. Epidemiology, patterns of care, and mortality for patients with acute respiratory distress syndrome in intensive care units in 50 countries. JAMA 2016;315(8):788-800.

11. Lellouche F, Taille S, Maggiore SM, Qader S, L'Her E, Deye N, et al. Influence of ambient and ventilator output temperatures on performance of heated-wire humidifiers. Am J Respir Crit Care Med 2004;170(10):1073-1079.

12. Garnier M, Quesnel C, Fulgencio JP, Degrain M, Carteaux G, Bonnet $\mathrm{F}$, et al. Multifaceted bench comparative evaluation of latest intensive care unit ventilators. Br J Anaesth 2015;115(1):89-98.

13. Jaecklin T, Morel DR, Rimensberger PC. Volume-targeted modes of modern neonatal ventilators: how stable is the delivered tidal volume? Intensive Care Med 2007;33:326-335.

14. Gillies D, Todd DA, Foster JP, Batuwitage BT. Heat and moisture exchangers versus heated humidifiers for mechanically ventilated adults and children. Cochrane Database Syst Rev 2017;(9):CD004711. 